\title{
Accuracy of reporting estimated blood loss in open repair of pelvic and acetabular fractures
}

\author{
Kavon Sharifi BS, Tanir Moreno MS, Samudani Dhanasekara PhD, Mia Ivos BA, Nicole Van Spronsen BS, \\ Caroline Chung BS, Cyrus Caroom MD, Robyn Richmond MD, Ariel Santos MD
}

\begin{abstract}
Background: Pelvic injuries after blunt trauma are often repaired with open reductioninternal fixation (ORIF), which can result in significant blood loss. We compared calculations of estimated perioperative blood loss (EPBL) against reported intraoperative blood loss (RIBL) by visual estimation to evaluate their accuracy during ORIF for pelvic fractures.

Methods: A retrospective observational study was conducted at a Level 1 trauma center. One hundred and forty-two patients requiring ORIF of acetabular and/or pelvic ring fracture between ages 18 to 89 years from March 1, 2017, to Feb. 28, 2019, were included. Estimated perioperative blood loss was calculated by the Gross Method. Statistical analyses were conducted using $R$ statistical software. Medians and inter-quartile ranges were used to summarize variables. A two-sample Wilcoxon signed rank test was performed. The agreement and reproducibility of RIBL and EPBL were examined with concordance correlation coefficients (CCC) and Bland-Altman plots.

Results: Median RIBL was $450 \mathrm{~mL}$ [250, 800], while median EPBL was $2142 \mathrm{~mL}$ [1213, 3607]. The median difference was $-1692 \mathrm{~mL}(p<0.001)$. The Bland-Altman plot showed consistent under-reporting in RIBL versus EPBL. A proportional bias $(p<0.001)$ indicated that the level of bias was not constant between levels of blood loss. The CCC between RIBL and EPBL was 0.076 [-0.016, 0.167]. Pearson's Correlation Coefficient was $0.213(p=0.011)$. RIBL and EPBL had a weak positive correlation.

Discussion: Blood loss during ORIF calculated by EPBL was significantly higher than RIBL. Our results suggest that blood loss reporting may be inaccurate in ORIF of pelvic fractures. These findings necessitate formulation of an accurate method permitting proper blood loss reporting.
\end{abstract}

Keywords: postoperative blood loss, surgical blood loss, orthopedic surgery, estimation

\section{INTRODUCTION}

Pelvic fractures occur at a high incidence of $9.3 \%$ after blunt trauma such as motor vehicle accidents, motorcycle injuries, falls and pedestrian injuries. ${ }^{1}$ Moreover, these injuries frequently result in significant

Corresponding author: Ariel Santos

Contact Information: Ariel.santos@ttuhsc.edu

DOI: 10.12746/swrccc.v9i41.893 blood loss. For instance, $38.5 \%$ of pelvic fractures require blood transfusions, and $16.6 \%$ require more than $2,000 \mathrm{~mL} .{ }^{1}$ Open reduction and internal fixation (ORIF) of pelvic and acetabular fractures result in significantly higher blood loss and transfusion requirements than closed techniques. ${ }^{2}$ However, decisions to perform open reduction versus closed reduction depends on many variables, including fracture type, patient factors, timing of the surgery, etc.

Although ORIF results in major blood loss, there is uncertainty in the literature as to the more significant 
causes of mortality in these patients. For instance, Schulman et al. and Holstein et al. found that pelvic ring fractures are an independent major risk factor for mortality in the blunt trauma population, and that more than $60 \%$ of deaths after pelvic ring fractures can be attributed to excessive blood loss. ${ }^{3,4}$ However, Demetriades et al. found that death due to exsanguination occurs in less than $1 \%$ of blunt trauma patients, and that mortality in this population is more commonly attributable to the high incidence of associated intra-abdominal injuries. ${ }^{1}$

These circumstances necessitate accurate measurements of relevant factors, such as blood loss, across all facets of this patient population. Dellinger et al. suggested that early prediction of a higher expected blood loss could initiate earlier volume resuscitation, thereby decreasing the risk of hypovolemic shock and mortality. ${ }^{5}$ This patient population is associated with a higher risk of blood loss, and an accurate estimation is crucial to ameliorate patient mortality and to increase proper reporting. In this study, we examined current predictive methods by evaluating the reported intraoperative blood loss (RIBL) during ORIF as compared to the estimated perioperative blood loss (EPBL) using calculations to gain insight into the contribution of blood loss during ORIF to overall blood loss in patients suffering from pelvic fractures. This will give valuable information in guiding the management of this vulnerable patient population both intra-operatively and postoperatively.

\section{Methods}

The study was approved by the Institutional Review Board at Texas Tech University Health Sciences Center, Lubbock, TX (TTUHSC IRB \#L19-033). A retrospective observational study was conducted at a Level 1 trauma center. One hundred and forty-two patients requiring ORIF of acetabular and/or pelvic ring fracture between ages 18 to 89 years old from March 1, 2017, to February 28, 2019, were included. Exclusion criteria included patients who underwent reduction and fixation via closed techniques.

A total of 142 records met the inclusion criteria. The mean age of the group was $41.94 \pm 20.44$ years
Table 1. Sample Characteristics in the Whole Sample

\begin{tabular}{|l|l|}
\hline Variable & Mean \pm SD or N (\%) \\
\hline Age (years) & $41.94 \pm 20.44$ \\
\hline Gender (Male) & $103(72.54 \%)$ \\
\hline Race $($ Caucasians) & $129(90.84 \%)$ \\
\hline BMI $\left(\mathrm{kg} / \mathrm{m}^{2}\right)$ & $29.17 \pm 8.57$ \\
\hline Diabetes mellitus & $14(9.86 \%)$ \\
\hline Hypertension & $33(23.24 \%)$ \\
\hline $\begin{array}{l}\text { Mechanism of injury } \\
\text { Motor vehicle crash }\end{array}$ & $87(61.27 \%)$ \\
Pedestrian struck & $12(8.45 \%)$ \\
Fall & $25(17.61 \%)$ \\
Other & $17(11.97 \%)$ \\
\hline
\end{tabular}

(range 8-92 years). The sample included 103 males $(72.54 \%)$ and 39 females (27.46\%). Fourteen (9.86\%) patients had diabetes, and $33(23.24 \%)$ had hypertension. The main mechanism of injury was blunt trauma $(\mathrm{n}=141,99.3 \%)$ (Table 1).

Calculation of estimated perioperative blood loss:

Volume loss was calculated using the modifications to the Gross Method as follows ${ }^{6}$ :

Calculated Volume Loss $=E B V \times(H 0-H F)$

Where:

EBV = estimated blood volume (calculated using Nadler's formula)

$\mathrm{H}_{0}=$ preoperative hematocrit on the day of ORIF $\mathrm{H}_{\mathrm{F}}=$ postoperative hematocrit 2-3 days after ORIF

The patient's estimated blood volume multiplied by the hematocrit will give the total red cell volume, so any change in red cell volume can therefore be calculated from the change in hematocrit. ${ }^{7}$

Estimated blood volume (EBV) was calculated using Nadler's formula as follows ${ }^{8}$ :

For men: $\mathrm{EBV}=\left(0.3669 \times\right.$ height $\left.^{3}\right)+(0.03219 \times$ weight) +0.6041 .

For women: $\mathrm{EBV}=\left(0.3561 \times\right.$ height $\left.^{3}\right)+(0.03308 \times$ weight) +0.1833

Where height is calculated in meters and weight is calculated in kilograms. 
Many patients required blood transfusion within the time period of this calculation (intra-operatively or up to three days postoperatively). This added RBC volume artificially raises the hematocrit. Therefore, this volume was added to the calculated volume loss in order to find the total volume loss. This final calculation represents the EPBL.

Descriptive statistics were expressed in mean \pm standard deviation (SD) (continuous variables) and in absolute and relative frequencies (categorical variables). The Shapiro-Wilk test of normality was conducted to assess the normality. This test was significantly different $(p<0.05)$ indicating that the distribution of the data was significantly different from normal distribution. Thus, non-parametric tests were conducted. Medians and inter-quartile ranges were used to summarize variables and a two-sample Wilcoxon signed rank test was performed to compare the group medians, considering-values less than 0.05 as significant.

Statistical analyses were conducted using R statistical software (version 3.5.3, The R Foundation, Vienna, Austria) and the distributions were examined visually. The concordance correlation coefficient (CCC) was measured to check agreement and reproducibility between reported perioperative blood loss and calculated perioperative blood loss. The agreement and reproducibility of RIBL and EPBL were examined by calculating concordance correlation coefficients (CCC) via a bootstrap approach employing 50 iterations and Bland-Altman plots using the agRee and blandr packages in $\mathrm{R}$ statistical software.

\section{RESULTS}

Reported intra-operative blood loss was underreported blood loss compared to EPBL. Median RIBL was $450 \mathrm{~mL}[250,800]$, while median EPBL was 2142 $\mathrm{mL}[1213,3607]$. The median difference was $-1692 \mathrm{~mL}$ $(p<0.001)$ (Figure 1). The Bland-Altman plot showed a consistent under-reporting (constant bias/error) in RIBL with regard to EPBL as well. Moreover, a Bland-Altman plot showed that there was a proportional bias $\left(R^{2}=\right.$ $0.5913, p<0.001$ ), indicating that the level of bias was not constant over different levels of blood loss (Figure 2). These results indicated that compared to EPBL, the degree of under-reporting of RIBL became larger as the blood loss became high. The CCC between RIBL and EPBL was $0.076[-0.016,0.167]$. Hence, there was poor agreement between the two methods. Pearson's Correlation Coefficient was $0.213(p=0.011)$. RIBL and EPBL had a weak positive correlation (Figure 3).

\section{Discussion}

Our results indicated that the agreement between the blood loss measured using visual estimation (RIBL) and EPBL seems to be poor. Currently, there is no gold standard calculation method to estimate perioperative blood loss, although various methods exist. ${ }^{9}$ Jaramillo et al. were unable to find an agreement between different methods to calculate blood loss. ${ }^{9}$ The documented discrepancies between various methods suggest that an amount of uncertainty exists, necessitating further inquiry. This study primarily focuses on comparing EPBL calculated by the Gross formula, a widely used method, with RIBL by visual estimation.

Reported intra-operative blood loss is often reported after surgery by visual estimation. Methods of visual estimation include the amount of blood in suction canisters, on the floor, on operating team members' gowns, as well as from soaked gauze and sponges. This comes with its own set of challenges, such as the presence of blood diluted by saline washes/other fluids as well as incompletely soaked sponges confounding the visual estimation. ${ }^{10,11}$ Another potential drawback to visual estimation is that blood loss tends to be overestimated when the amount of hemorrhaging is small and underestimated with larger amounts of hemorrhage. ${ }^{12}$ Our results indicated that there is significant under-reporting at higher volumes of blood loss. Hence, this presents a problem of its own in our patient population, in which hemorrhaging tends to be greater when compared to many other surgical populations.

The large discrepancy we found between blood loss measured using two methods as well as the consistent under-reporting of RIBL compared to EPBL suggests flaws in one or both methods. Pelvic fractures are often complicated by retroperitoneal 


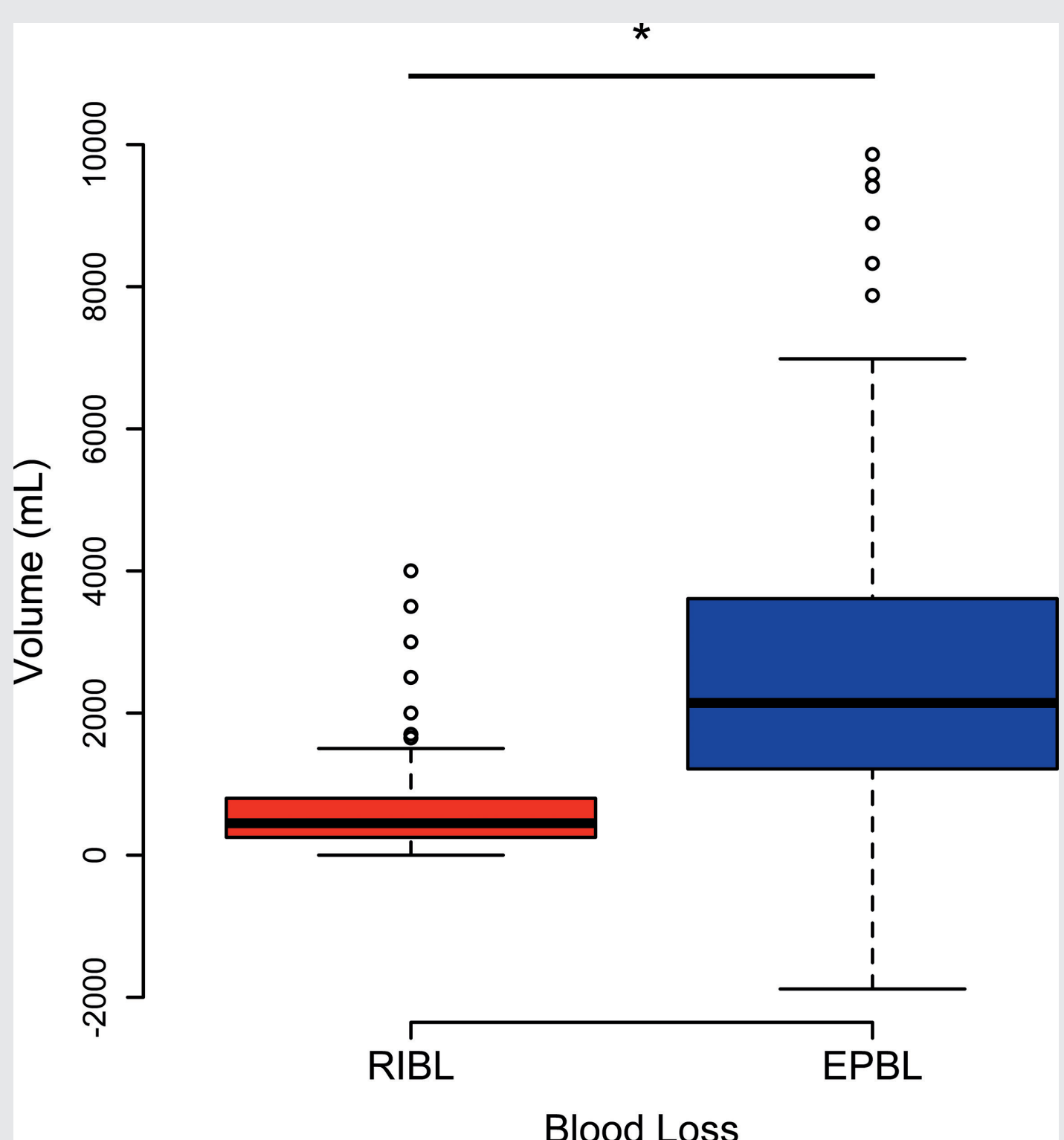

Figure 1. Box plot of reported intraoperative blood loss (RIBL) compared to estimated perioperative blood loss (EPBL), demonstrating the large difference in median blood loss. EPBL had a wider range than RIBL.

bleeding that may not be accurately detected in the first place, resulting in frequent "hidden bleeds." ${ }^{13}$ Many patients may have other associated injuries that can account for higher EPBL. These extraneous bleeds will, based on the nature of the formulae, significantly increase the EPBL, especially in pelvic fracture populations.
There is a challenge to definitively make this assertion because this population may be hemorrhaging from many sources due to the initial blunt trauma, introducing a potential skew to the results and a source of limitation for EPBL. A modification to increase the reliability of these results may be to increase the study sample size. Intraoperative 


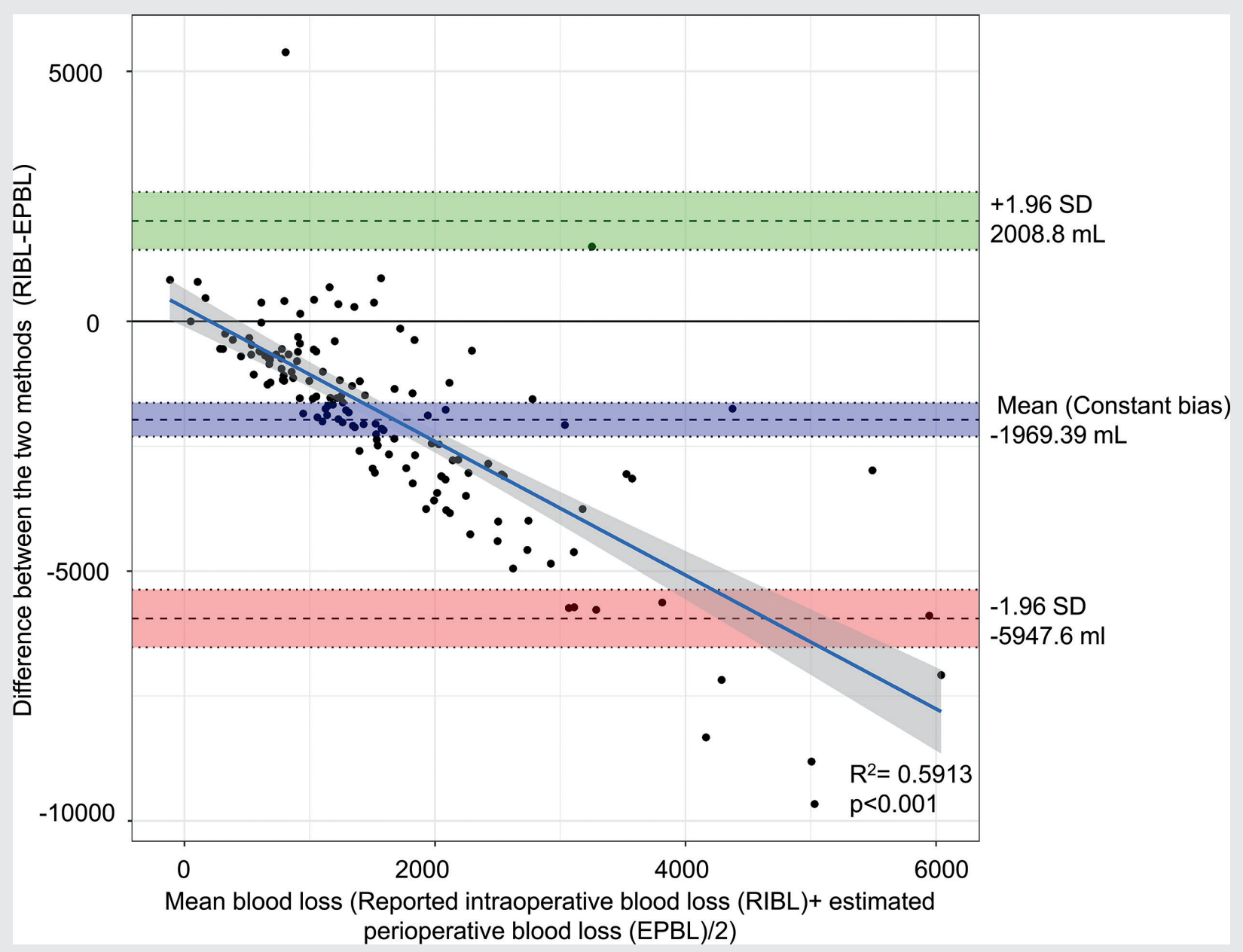

Figure 2. The Bland-Altman plot shows a proportional difference in variability between each method. There is constant coefficient of variation across the range of blood volumes. The blue line represents the regression line of proportional bias $(y=-1.3 x+270)$. Dashed-lines represent the mean (constant bias) and color shades indicate $95 \%$ confidence intervals.

transfusions can also alter the EPBL which is not taken into account in this study.

Estimated perioperative blood loss calculation by the Gross method and RIBL calculation by visual estimation show little agreement, and neither may necessarily be a true representation of intra-operative blood loss. As previously discussed, visually estimated RIBL tends to underestimate in situations with greater blood loss. Additional research is necessary to provide an accurate method of estimating blood loss in the operating room, particularly in the pelvic fracture population. This method must account for both the significant non-surgical bleeding in this particular population as well as the aforementioned flaws in visual estimation. 


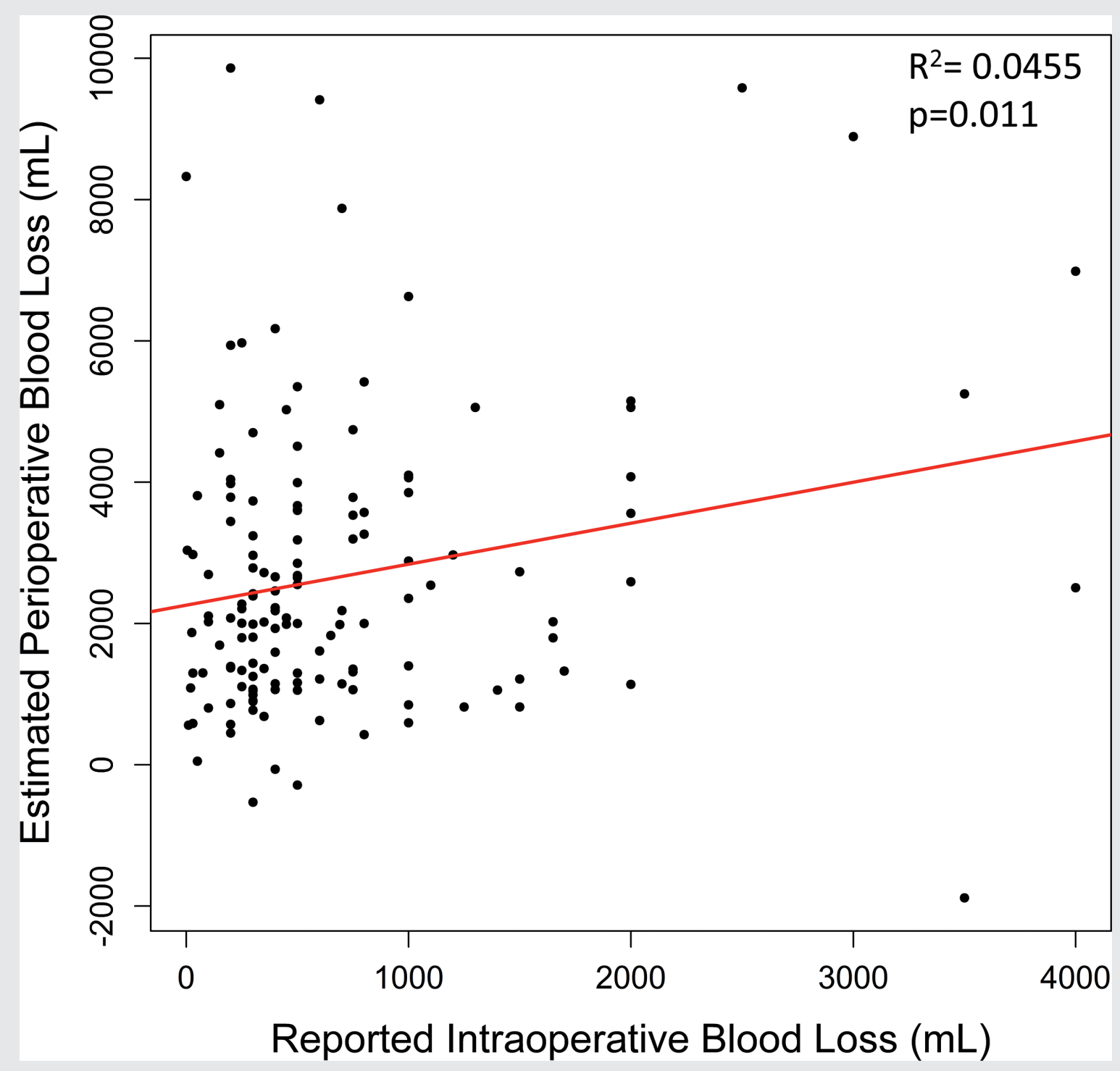

Figure 3. Correlation between reported intraoperative blood loss (RIBL) to the estimated perioperative blood loss $(\mathrm{EPBL})$. The red line represents the regression line $\left(\mathrm{R}^{2}=0.046, \mathrm{p}=0.011\right)$.

Table 2. Summary of Median and Inter-Quartile Ranges

\begin{tabular}{|l|l|l|l|l|l|}
\hline & $\begin{array}{l}\text { Median of Reported } \\
\text { Intraoperative Blood } \\
\text { Loss [Q1, Q3] } \mathbf{( m L )}\end{array}$ & $\begin{array}{l}\text { Median of Calculated } \\
\text { Perioperative Blood Loss } \\
\text { [Q1, Q3] }(\mathbf{m L})\end{array}$ & $\begin{array}{l}\text { Median Difference } \\
(\mathbf{m L})\end{array}$ & W Statistics* & P Value \\
\hline $\begin{array}{l}\text { Blood } \\
\text { Loss }\end{array}$ & $450[250,800]$ & $2142[1213,3607]$ & -1692 & 17737 & $<0.001$ \\
\hline
\end{tabular}

* two-sample Wilcoxon signed rank test. 
Article citation: Sharifi, K, Moreno T. Dhanasekara S, Ivos M, Van Spronsen N, Chung C, Caroom C, Richmond R, Santos A. Accuracy of reporting estimated blood loss in open repair of pelvic and acetabular fractures. The Southwest Respiratory and Critical Care Chronicles 2021;9(41):1-7

From: The School of Medicine (KS, TM, MI, NVS, CC), Department of Surgery (SD, RR, AS), and Department of Orthopedics (CC), Texas Tech University Health Sciences Center, Lubbock, Texas

Submitted: $7 / 5 / 2021$

Accepted: 10/9/2021

Reviewer: Michael Phy DO

Conflicts of interest: none

This work is licensed under a Creative Commons

Attribution-ShareAlike 4.0 International License.

\section{REFERENCES}

1. Demetriades D, Karaiskakis M, Toutouzas K, et al. Pelvic fractures: epidemiology and predictors of associated abdominal injuries and outcomes. J Am Coll Surg 2002;195(1):1-10.

2. Elzohairy MM, Salama AM. Open reduction internal fixation versus percutaneous iliosacral screw fixation for unstable posterior pelvic ring disruptions. Orthop Traumatol Surg Res 2017;103(2):223-227.

3. Schulman JE, O'Toole RV, Castillo RC, et al. Pelvic ring fractures are an independent risk factor for death after blunt trauma. J Trauma 2010;68(4):930-934.
4. Holstein JH, Culemann U, Pohlemann T. Working Group Mortality in Pelvic Fracture Patients. What are predictors of mortality in patients with pelvic fractures? Clin Orthop Relat Res 2012;470(8):2090-2097.

5. Dellinger RP, Levy MM, Rhodes A, et al. Surviving Sepsis Campaign: international guidelines for management of severe sepsis and septic shock, 2012. Intensive Care Med 2013; 39(2):165-228.

6. Gross JB. Estimating allowable blood loss: corrected for dilution. Anesthesiology 1983;58(3):277-280.

7. Bourke DL, Smith TC. Estimating allowable hemodilution. Anesthesiology 1974;41(6):609-612.

8. Nadler SB, Hidalgo JH, Bloch T. Prediction of blood volume in normal human adults. Surgery 1962;51(2):224-232.

9. Jaramillo S, Montane-Muntane M, Capitan D, et al. Agreement of surgical blood loss estimation methods. Transfusion 2019;59(2):508-515.

10. Meiser A, Casagranda O, Skipka G, et al. Quantification of blood loss. How precise is visual estimation and what does its accuracy depend on?. Anaesthesist 2001;50(1):13-20.

11. Ulusoy A, Demiroz S, Erdem S. How accurate is visual estimation of perioperative blood loss in adolescent idiopathic scoliosis surgery? Acta Orthop Traumatol Turc 2018; 52(4):267-271.

12. Donham B, Frondozo R, Petro M, et al. Clinical Image: Visual estimation of blood loss. J Spec Oper Med. 2017; 17(1):68-71.

13. Mohanty K, Musso D, Powell JN, et al. Emergent management of pelvic ring injuries: an update. Can J Surg 2005;48(1): 49-56. 